\title{
Development of Superconducting Tunnel Junction Detectors as a far-infrared single photon detector for neutrino decay search
}

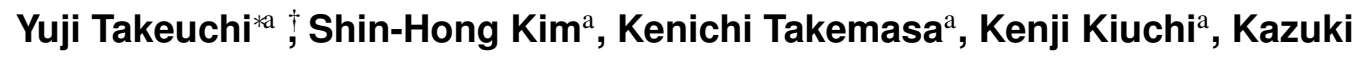
Nagata $^{a}$, Kota Kasaharaa ${ }^{a}$, Takuya Okudaira ${ }^{a}$, Tatsuya Ichimura ${ }^{a}$, Masahiro Kanamaru $^{\mathrm{a}}$, Kouya Moriuchi ${ }^{\mathrm{a}}$, Ren Senzaki ${ }^{\mathrm{a}}$, Hirokazu Ikeda ${ }^{\mathrm{b}}$, Shuji Matsuura ${ }^{\mathrm{b}}$, Takehiko Wada $^{\mathrm{b}}$, Hirokazu Ishino ${ }^{\mathrm{c}}$, Atsuko Kibayashi ${ }^{\mathrm{c}}$, Satoru Mima ${ }^{\mathrm{d}}$, Takuo Yoshida $^{\mathrm{e}}$, Shota Komura ${ }^{\mathrm{e}}$, Keisuke Orikasa ${ }^{\mathrm{e}}$, Ryuta Hirose ${ }^{\mathrm{e}}$, Yukihiro Kato ${ }^{\mathrm{f}}$, Masashi Hazumi $^{\text {g }}$, Yasuo Arai ${ }^{\mathrm{g}}$, Erik Ramberg ${ }^{\mathrm{h}}$, Jonghee Yoo $^{\mathrm{h}}$, Mark Kozlovsky ${ }^{\mathrm{h}}$, Paul Rubinov $^{\text {h }}$, Dmitri Sergatskov ${ }^{\text {h }}$, Soo-Bong Kim ${ }^{\mathrm{i}}$

${ }^{a}$ University of Tsukuba, 1-1-1 Ten-nodai, Tsukuba, Ibaraki 305-8571, Japan

${ }^{\mathrm{b}}$ Institute of Space and Astronautical Science, JAXA, 3-1-1 Yoshinodai, Chuo-ku, Sagamihara, Kanagawa 252-5210, Japan

${ }^{\mathrm{c} O k a y a m a}$ University, 1-1-1 Tsushima-naka, Kita-ku, Okayama 700-8530, Japan

${ }^{\mathrm{d}}$ RIKEN, 2-1 Hirosawa, Wako, Saitama 351-0198, Japan

e University of Fukui 3-9-1 Bunkyo, Fukui 910-8507, Japan

${ }^{\mathrm{f}}$ Kinki University 3-4-1 Kowakae, Higashiosaka, Osaka 577-8502, Japan

${ }^{\mathrm{g}}$ High Energy Accelerator Research Organization, KEK, 1-1 Oho, Tsukuba, Ibaraki 305-0801 Japan

${ }^{\mathrm{h}}$ Fermi National Accelerator Laboratory Batavia, IL 60510-5011, USA

${ }^{i}$ Seoul National University, 1 Gwanak, Gwanak-ro, Gwanak-gu, Seoul, 151-742, Korea

\begin{abstract}
We present the development of Superconducting Tunnel Junction (STJ) detectors as a far-infrared single photon detector motivated by application to a search for the radiative decay of cosmic background neutrino. The photon energy spectrum from the neutrino radiative decays is expected to have a sharp edge at high energy end in a far-infrared region ranging from $14 \mathrm{meV}$ to $25 \mathrm{meV}$ (from $50 \mu \mathrm{m}$ to $90 \mu \mathrm{m}$ in wavelength). Thus, the detector is required to measure photon-byphoton energies with sufficiently high resolution to identify the edge structure. Detectors consisting of an array of niobium/aluminum STJ (Nb/Al-STJ) pixels with a diffraction grating and STJ using hafnium (Hf-STJ) are under consideration. Each Nb/Al-STJ pixel is capable of detecting single photons in the far-infrared region. The diffraction grating creates a variation in the photon wavelength across the pixel array. Hf-STJ is expected to have $2 \%$ energy resolution for single photon of $25 \mathrm{meV}$ due to very small gap energy of hafnium.
\end{abstract}

Technology and Instrumentation in Particle Physics 2014,

2-6 June, 2014

Amsterdam, the Netherlands

\footnotetext{
* Speaker.

†takeuchi@hep.px.tsukuba.ac.jp
} 


\section{Introduction}

Since the mixings among three flavors of neutrinos were established and their squared mass differences among generations were found to be non-zero by various experiments, one has been convinced that neutrino can decay into lighter neutrino with photon. Figure 1 illustrates typical radiative neutrino decay diagrams in the standard model (SM) as well as in the Left-Right Symmetric model (LRSM). The radiative decay process is related to the transition magnetic moment

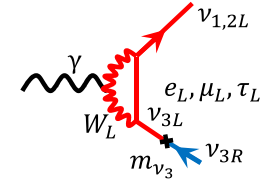

$S M: S U(2)_{L} \times U(1)_{Y}$

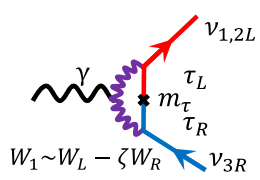

LRS: $S U(2)_{L} \times S U(2)_{R} \times U(1)_{B-L}$

Figure 1: Typical diagrams of radiative neutrino decay in the standard model (left) and in LRSM (right). In the LRSM, mostly the charged tau contributes to the loop. The subscripts of $\mathrm{L}(\mathrm{R})$ in the particles indicate left(right)-handed chiralities.

term of neutrino. In the standard model, however, the transition magnetic moment term is highly suppressed by the small mass of neutrino $m_{v}$, through which left- and right-handed chiralities can couple. The process is further suppressed by Glashow-Iliopoulos-Maiani (GIM) mechanism due to similar contributions from $e, \mu$, and $\tau$ to the loop. Because of these suppressions, the lifetime in the standard model is predicted to be in the order of $10^{43}$ years for the heaviest neutrino $v_{3}$ [1], while the current experimental lower limit on the lifetime is only given to be in the order of $10^{12}$ years [3]. On the other hand, in the left right symmetric model, if we assume there is only a small mixing between $W_{\mathrm{L}}$ and $W_{\mathrm{R}}$ bosons which can couple only to left- and right-handed chiralities, respectively, it makes the lifetime much shorter [2], since left- and right-handed chiralities can couple through much larger $\tau$ mass in the loop dominantly, and thus there is no GIM suppression, either. The lifetime calculation based on values from recent experiments is performed in Ref. [3]. If we suppose the mixing angle to be $|\zeta|<0.02$ and the mass of $v_{3}$ to be $m_{3}=50 \mathrm{meV}$, the lifetime of $v_{3}$ is predicted to be $\tau_{v_{3}}<1.5 \times 10^{17}$ years ${ }^{1}$ in LRSM.

Hence, we have proposed to search for the neutrino radiation decay using the cosmic background neutrino $(\mathrm{C} v \mathrm{~B})$, which is predicted to exist with the density of $\rho_{v} \sim 110 / \mathrm{cm}^{3}$ per generation and with temperature of $T_{v}=1.95 \mathrm{~K}$ at present.The experiment is motivated by the direct detection of $\mathrm{CvB}$, the direct detection of the transition magnetic moment of neutrino, and also it turns out to be connected to the determination of neutrino mass from the photon energy. The photon energy in the neutrino radiative decay $v_{3} \rightarrow v_{1,2}+\gamma$ is described as $E_{\gamma}=\left(m_{3}^{2}-m_{1,2}^{2}\right) / 2 m_{3}$ in the rest frame of the parent neutrino, where $m_{i}$ denotes the neutrino mass of the $i$-th generation. The $v_{3}$ mass is constrained by $\Delta m_{23}^{2} \sim 2.4 \times 10^{-3} \mathrm{eV}^{2}$ and $\sum m_{i}<0.23 \mathrm{eV}$ [5] obtained from various oscillation measurements and cosmic microwave background (CMB) in combination with baryon acoustic oscillation (BAO) measurements, respectively, and consequently, is likely in the range of

\footnotetext{
${ }^{1}$ In the neutrino lifetime calculation in LRSM in Ref. [3], Maki-Nakagawa-Sakata (MNS) matrix elements were not correctly considered. For $|\zeta|<0.01$ that was adopted in the paper, the lifetime must be $\tau_{v_{3}}<5.6 \times 10^{17}$ years.
} 
$50 \mathrm{meV}<m_{3}<87 \mathrm{meV}$. If $m_{3}=50 \mathrm{meV}$ is assumed, the photon energy is $25 \mathrm{meV}$ (50 $\mu \mathrm{m}$ in wavelength).

The more realistic photon energy spectrum we observe is expected to have the sharp edge at $25 \mathrm{meV}$ and the tail in the lower energy (longer wavelength) side because of the red-shift effect $z=\Delta \lambda / \lambda$ for the distant neutrino decays. The contribution to the surface brightness $I_{v}$ from the neutrino radiative decay as a function of wavelength is predicted to be

$$
I_{v}=\frac{\rho_{v} c h}{4 \pi \tau_{v} H_{0}}\left[\left(\frac{\lambda}{\lambda_{0}}\right)^{3} \Omega_{M}+\Omega_{\Lambda}\right]^{-1 / 2}=\frac{2.5 \times 10^{12} \mathrm{yr}}{\tau_{v}}\left[0.3\left(\frac{\lambda}{\lambda_{0}}\right)^{3}+0.7\right]^{-1 / 2} \mathrm{MJy} / \mathrm{sr},
$$

where $\lambda_{0}$ means the wavelength of the photon from the decay at $z=0, H_{0}$ is the Hubble constant of $67.80 \pm 0.77 \mathrm{~km} / \mathrm{s} / \mathrm{Mpc}$ [5], and $\Omega_{M}$ and $\Omega_{\Lambda}$ are supposed the matter density of 0.3 and the cosmological constant of 0.7 , respectively. The effect from thermal motions of the relativistic Fermi-Dirac distribution of $\mathrm{C} v \mathrm{~B}$ at $T_{v}=1.95 \mathrm{~K} \times(1+z)$ is also considered in the plot. Backgrounds to the photon from the neutrino decay are the overwhelming zodiacal emission (ZE) and cosmic infrared backgrounds (CIB). Figure 2 shows the contributions to the surface brightness $I_{v}$ from the neutrino decay on the assumptions of $\tau_{v_{3}}=1 \times 10^{14}$ years and $5 \times 10^{12}$ years, overlaid on the summary plot including the various foregrounds and CIB measurements in the infrared region. The plot shows the brightness from the $\mathrm{C} v \mathrm{~B}$ radiative decay with $10^{12}$ year lifetime is at same level

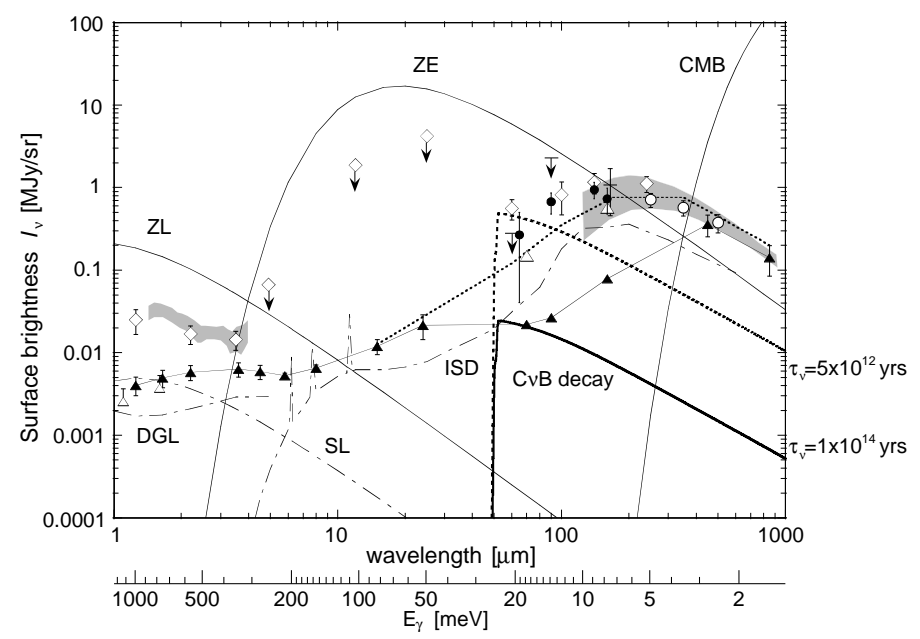

Figure 2: Contribution from the $\mathrm{C} v \mathrm{~B}$ radiative decays to the surface brightness $I_{v}$ overlaid on the summary plot with the various foregrounds and CIB measurements together with AKARI ADF-S results (filled circles) taken from Fig. 1 and from Fig. 13 in Ref. [4]. The components from the $\mathrm{C} v \mathrm{~B}$ radiative decays are shown by the solid curve for $\tau_{v_{3}}=1 \times 10^{14}$ years and the dotted curve for $\tau_{v_{3}}=5 \times 10^{12}$ years. In both cases, $m_{v_{3}}=50 \mathrm{meV}$ is assumed, and thermal motions of $\mathrm{C} v \mathrm{~B}$ at $T_{v}=1.95 \mathrm{~K} \times(1+z)$ are also considered.

as CIB, which implies the lifetime of the order of $10^{12}$ years is already ruled out as described in Ref. [3].

\section{Experiment on search for neutrino radiative decay}

We need highly precise measurement of the photon energy spectrum around $50 \mu \mathrm{m}$ in wave- 
length for better sensitivity to the lifetime measurement. While the final goal of our proposal is sensitivity to the lifetime in the order of $10^{17}$ years, we aim at sensitivity in the order of $10^{14}$ years first. In case of $\tau_{v}=10^{14}$ years, the brightness from the the $\mathrm{C} v \mathrm{~B}$ radiative decay is 2.5 order smaller than the zodiacal emission (ZE). Therefore, the requirements for the detector are to obtain continuous spectrum of photon energy around $50 \mu \mathrm{m}$ far-infrared photon, and energy measurement for a single photon with better than $2 \%$ resolution to identify the sharp edge in the spectrum. Furthermore, the detector is supposed to be loaded on a rocket and/or satellite.

Our choice for the detector is a Superconducting Tunnel Junction (STJ) detector [6]. STJ is a Josephson junction device, which has thin superconductor-insulator-superconductor layers. A bias voltage is applied across the junction, and a photon absorbed in the superconductor layer breaks Cooper pairs and creates tunnel current of quasi-particles proportional to the photon energy with better energy resolution thanks to much smaller superconducting gap energy than the semiconductor. Two kinds of STJs are under development for the experiment. One is an array of niobium/aluminum-STJ (Nb/Al-STJ) pixels in combination with a diffraction grating, which is supposed to be adopted in a rocket experiment, and the other is a hafnium-STJ (Hf-STJ) for a future experiment. Statistical fluctuation in the number of quasi-particles created by the photon energy deposit determines STJ energy resolution. Thus, smaller superconducting gap energy $(\Delta)$ yields better energy resolution. As for Nb/Al-STJ, it is well-established [7]. However, since the gap energy of niobium is about $1.55 \mathrm{meV}$, number of quasi-particles to a $25 \mathrm{meV}$ photon is insufficient for $2 \%$ energy resolution. Instead, we employ Nb/Al-STJ as a photon counting device. Several groups have reported single photon detections with STJ up to $\lambda \sim 2 \mu \mathrm{m}$ in wavelength [8]. As for Hf-STJ, the gap energy of hafnium is about $20 \mu \mathrm{eV}$ and number of quasi-particles is sufficient to achieve $2 \%$ energy resolution.

As mentioned, we are planning a rocket experiment using $\mathrm{Nb} / \mathrm{Al}-\mathrm{STJ}$ array with grating aiming at the $\mathrm{C} v \mathrm{~B}$ decay search with sensitivity of $10^{14}$ year for the lifetime. We expect about 200 second measurement after the launch at altitude from 200 to $300 \mathrm{~km}$. The observatory has a $15 \mathrm{~cm}$ diameter and $1 \mathrm{~m}$ focal length telescope which will be cooled below $4 \mathrm{~K}$. We employ a diffraction grating covering from 40 to $80 \mu \mathrm{m}$ and an array of $\mathrm{Nb} / \mathrm{Al}-\mathrm{STJ}$ pixels of 50 along to the wavelength direction by 8 along to a spatial direction, where each Nb/Al-STJ pixel works as a single-photon counting detector with $100 \times 100 \mu \mathrm{m}^{2}$ sensitive area. If we assume the ideal conditions for all of them, the photon counting rate per one pixel is expected $340 \mathrm{~Hz}$ for the foreground and CIB emissions with these instruments, and the measurement for $200 \mathrm{~s}$ will yield $0.55 \mathrm{M}$ events to the spatial 8 pixels at $\lambda_{\gamma}=50 \mu \mathrm{m}$. This gives $0.13 \%$ precision for the surface brightness measurement $\left(\delta I_{v} \sim 10 \mathrm{kJy} / \mathrm{sr}\right)$ in each wavelength bin. Comparing to the precision, the surface brightness from the $\mathrm{C} v \mathrm{~B}$ decay on the assumption of $\tau_{v}=10^{14}$ years is expected $\sim 25 \mathrm{kJy} / \mathrm{sr}$. Thus, we will observe a sharp "ramp" at some wavelength, at which the spectrum has difference in level at $2.5 \sigma$ significance in each wavelength bin, that yields sufficient significance for us to detect the $\mathrm{C} v \mathrm{~B}$ radiative decay.

\section{Development of $\mathrm{Nb} / \mathrm{Al}-\mathrm{STJ}$ as a single photon counting device}

During the single photon counting at $\lambda_{\gamma}=50 \mu \mathrm{m}$ signal-to-noise ratio greater than $10^{3}$ is required. This requires the leak current of the $\mathrm{Nb} / \mathrm{Al}-\mathrm{STJ}$ to be less than $0.1 \mathrm{nA}$, considering the shot noise from the leak current. We have achieved 2 nA leak current so far as shown in Fig. 3, 
using a Nb/Al-STJ with $40 \mu \mathrm{m}^{2}$ junction size. The leak current depends on temperature as well as junction size. We measured the temperature dependence of the leak current down to $T=0.8 \mathrm{~K}$ or below for two $\mathrm{Nb} / \mathrm{Al}$-STJs with different junction sizes using a dilution refrigerator. The resulting plot is shown in Fig. 4, implying a potential for less leak current for a STJ with smaller junction size. Hence, we are testing a $\mathrm{Nb} / \mathrm{Al}-\mathrm{STJ}$ with a smaller junction size currently. We also found temperature below $0.9 \mathrm{~K}$ is required for the $\mathrm{Nb} / \mathrm{Al}-\mathrm{STJ}$ operation. We, thus, consider a ${ }^{3} \mathrm{He}$ sorption or an adiabatic demagnetization refrigerator (ADR) for the rocket experiment.

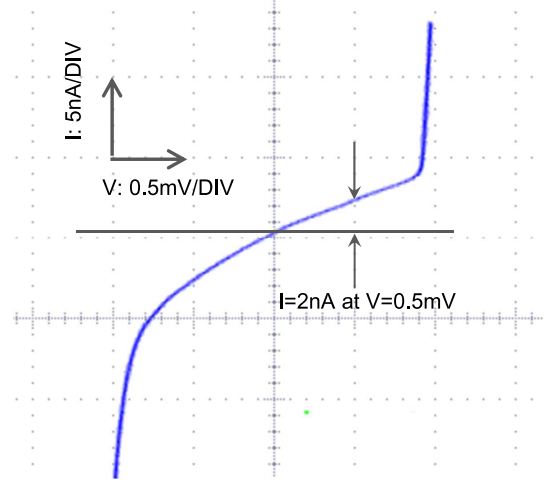

Figure 3: $I-V$ curve of a $\mathrm{Nb} / \mathrm{Al}-\mathrm{STJ}$ with $40 \mu \mathrm{m}^{2}$ junction size, measured at temperature of $0.4 \mathrm{~K}$ in a magnetic field, using $1 \mathrm{M} \Omega$ reference resistance for the current measurement.

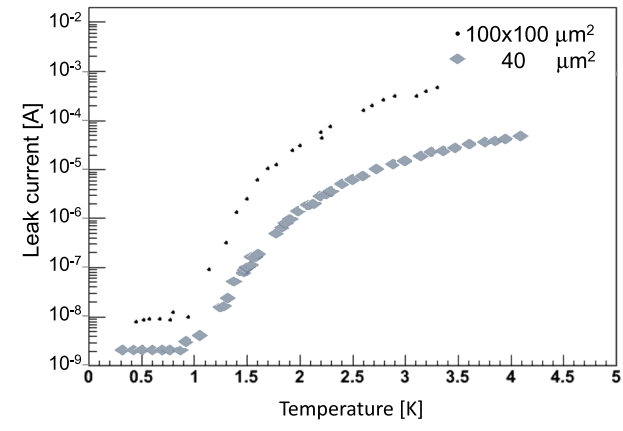

Figure 4: Temperature dependence on leak current measured for a Nb/Al-STJ with the junction size of $100 \times 100 \mu \mathrm{m}^{2}$ (dot), and of $40 \mu \mathrm{m}^{2}$ (filled diamond). The leak currents are observed to bottom out around $10 \mathrm{nA}$ and $2 \mathrm{nA}$, respectively, at temperature below $1 K$.

We tested the response to near-infrared $(\lambda=1.3 \mu \mathrm{m})$ photons at temperature of $1.8 \mathrm{~K}$ for a $\mathrm{Nb} / \mathrm{Al}$-STJ with $100 \times 100 \mu \mathrm{m}^{2}$ junction size, using the setup shown in Fig. 5. The bias current is supplied to the STJ through a resistance $R_{\text {ref }}$ from the constant voltage source. Since we use the much lager $R_{\text {ref }}$ than the STJ dynamic resistance at the nominal operating point, the STJ response to the photon pulse illumination is observed as a drop in the voltage difference across the STJ junction. We observed the response to photons with $\sim 1 \mu$ s response time as shown in Fig. 6, and we estimated the number of detected photons in the STJ assuming the dispersion of the signal pulse height distribution represents the statistical fluctuation in the number of detected photons. The observed signal response is found to correspond to a 40-photon detection.

We also tested the response to visible $(\lambda=465 \mathrm{~nm})$ photon illumination at single photon level for a Nb/Al-STJ with $4 \mu \mathrm{m}^{2}$ junction size at $T=1.8 \mathrm{~K}$. Figure 7 shows the pulse height distributions for the signal response to photon illumination and the pedestal. the pedestal distribution is taken using off-timing gate simultaneously with the signal distribution. The pedestal distribution is scaled to the estimated contribution from 0 photon in the signal distribution which is obtained as described below. The signal distribution is apparently dominated by the pedestal noise. Assuming a Poisson distribution convoluted with Gaussian which has same $\sigma$ as the pedestal noise, we consequently obtain the following projection for the signal distribution:

$$
f(x ; \mu)=N_{\text {tot }} \sum_{n} \frac{\mu^{n} \exp (-\mu)}{n !} \cdot \frac{1}{\sqrt{2 \pi} \sigma_{\mathrm{ped}}} \exp \left[-\frac{\left\{x-n\left(\frac{M}{\mu}\right)\right\}^{2}}{2 \sigma_{\mathrm{ped}}^{2}}\right],
$$




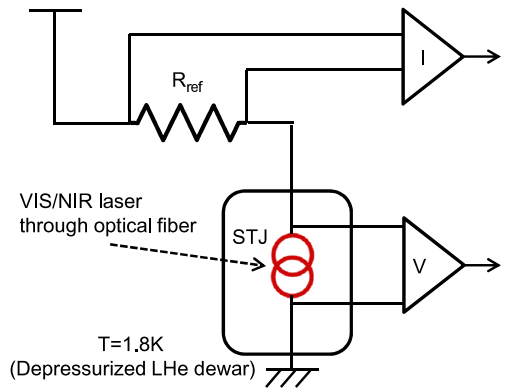

Figure 5: Setup for measurement of STJ response to the laser pulse illumination. the STJ is mounted on the cryostat in the Dewar where liquid helium is depressurized by a rotary pump to reach temperature below $1.8 \mathrm{~K}$. Visible or near-infrared laser pulses are transmitted via an optical fiber into the Dewar and emitted onto the STJ. The bias current is applied to the STJ through a resistance $R_{\text {ref }}$, and the voltage of the STJ is read out with a high impedance differential amplifier at the room temperature.

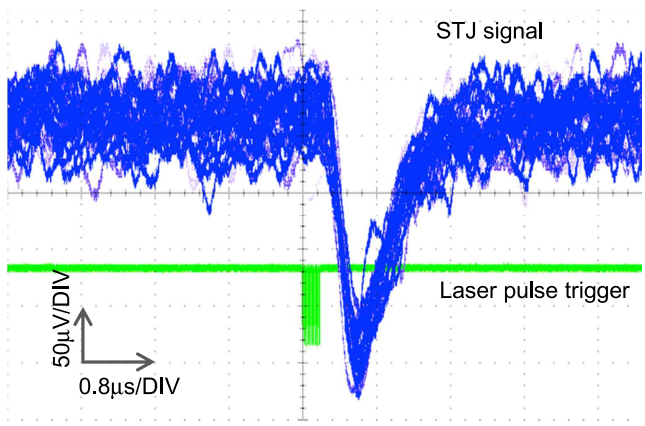

Figure 6: Response to near-infrared laser $(\lambda=$ $1.3 \mu \mathrm{m})$ pulses at $T=1.8 \mathrm{~K}$ for Nb/Al-STJ with $100 \times 100 \mu \mathrm{m}^{2}$ junction size, measured with an oscilloscope. 10 laser pulses are emitted in $200 \mathrm{~ns}$ triggered by the external pulses which are also shown in the picture. The signal response is observed as a drop in the STJ voltage, and found the typical response time is in the order of $1 \mu \mathrm{s}$.

where $x$ denotes a pulse height with respect to the mean of pedestal distribution, $\mu$ is the number of the detected photons, $N_{\text {tot }}$ is for normalization to the number of events, $\sigma_{\text {ped }}$ is sigma of the pedestal distribution, and $M$ is the mean of signal distribution. The signal distribution is fitted to the function, being $\mu$ as a fitting parameter and we obtained number of detected photons to be $0.93_{-0.14}^{+0.19}$.

To reduce the readout noise, we are developing several cold pre-amplifiers which can be placed on the cold stage very close to the STJ, expecting the reduction of noise picked up by the cable from the STJ to the pre-amplifier as well as thermal noise in the pre-amplifier. One of the promising candidates is SOI-STJ, where we process a STJ directly on the Silicon-On-Insulator (SOI) wafer in which a pre-amplifier circuit is implemented. The detail on the SOI-STJ development is described elsewhere [9].

\section{Development of Hf-STJ as a single photon calorimetry device}

We succeeded in the observation of DC Josephson current with a prototype Hf-STJ sample in 2011 [10]. Figure 8 shows $I-V$ curve of a Hf-STJ sample with $200 \times 200 \mu \mathrm{m}^{2}$ junction size produced in 2012, measured with the dilution refrigerator. We can observe the DC Josephson current at $V=0$, which is suppressed by magnetic field as expected. However, to operate the sample as a single photon detector with $2 \%$ energy resolution, we require the leak current in the order of $1 \mathrm{pA}$ or below, while the sample shows the leak current of about $50 \mu \mathrm{A}$. Therefore, we need much improvement in the leak current for Hf-STJ at present.

We illuminated visible $(\lambda=465 \mathrm{~nm})$ laser light to a Hf-STJ sample with $100 \times 100 \mu \mathrm{m}^{2}$ junction size. Figure 9 shows the $I-V$ curve with and without the continuous laser illumination. We observed a significant increase by $\sim 10 \mu \mathrm{A}$ caused by the tunnel current of quasi-particles in response to the photon illumination in the Hf-STJ dynamic resistance region. 


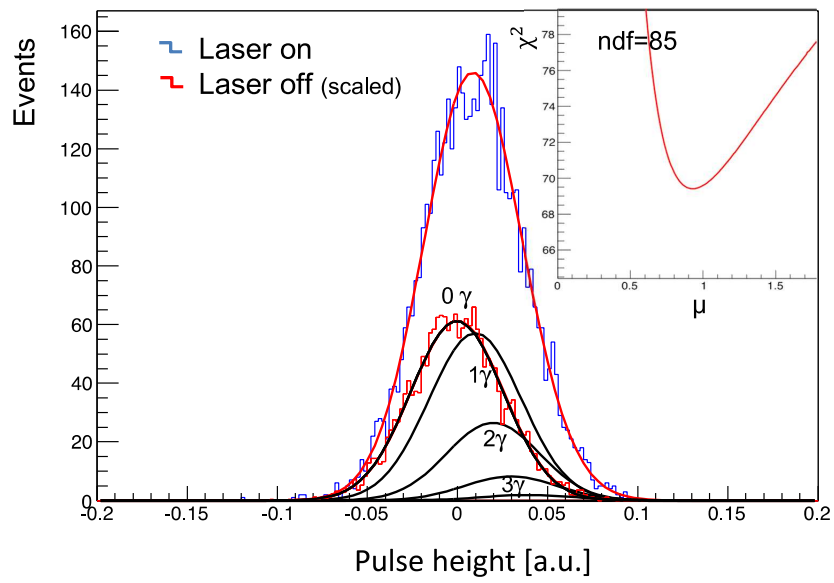

Figure 7: Pulse height distribution of a Nb/Al-STJ with $4 \mu \mathrm{m}^{2}$ junction size in response to two visible laser $(\lambda=465 \mathrm{~nm})$ pulses measured at $T=1.8 \mathrm{~K}$. The pedestal distribution is taken simultaneously, and shown in the plot, scaled to the estimated 0 photon contribution in the signal distribution. The signal distribution is fitted to a curve predicted by assuming a Poisson distribution convoluted with Gaussian which has same sigma as the pedestal distribution. The $\chi^{2}$ of the fitting as a function of assumed number of detected photons is also superimposed in the figure. The number of detected photons is measured to be $0.93_{-0.14}^{+0.19}$. Estimated contributions from $n$ photons are shown together.

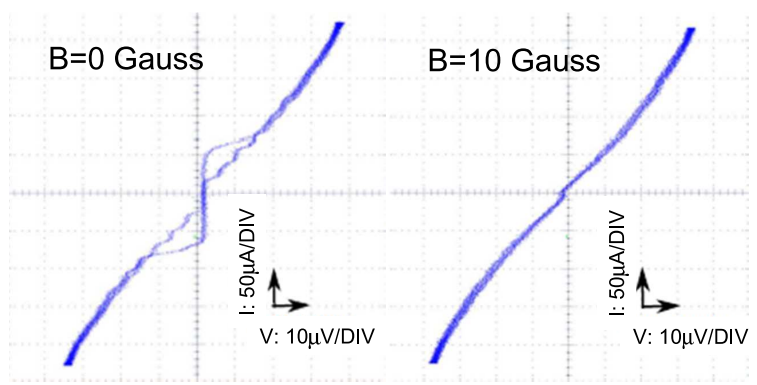

Figure 8: $I$ - $V$ curves of a Hf-STJ sample with $200 \times 200 \mu \mathrm{m}^{2}$ junction size, without (left) and with (right) magnetic field, measured at $T=80 \sim 177 \mathrm{mK}$. DC Josephson current can be seen clearly in the $I-V$ curve without magnetic field and it is suppressed in the $I-V$ curve with magnetic field. The leak current is found to be about $50 \mu \mathrm{A}$ at $V=10 \mu \mathrm{V}$.

\section{Summary}

We propose an experiment to search for neutrino radiative decay in cosmic background neutrino. We are developing a detector to measure single photon energy with better than $2 \%$ resolution for $E_{\gamma}=25 \mathrm{meV}$. A Nb/Al-STJ array with diffraction grating and a Hf-STJ are being considered for the detector. We observed the $\mathrm{Nb} / \mathrm{Al}-\mathrm{STJ}$ response to visible light at single photon level as the first milestone, although the signal from the $\mathrm{Nb} / \mathrm{Al}-\mathrm{STJ}$ is currently dominated by the readout noise. We have confirmed the superconductor-insulator-superconductor structure in our Hf-STJ prototypes and observed a tunnel current of quasi-particles in response to visible light illumination. However, much improvement in reduction of the leak current is required for a practical application 


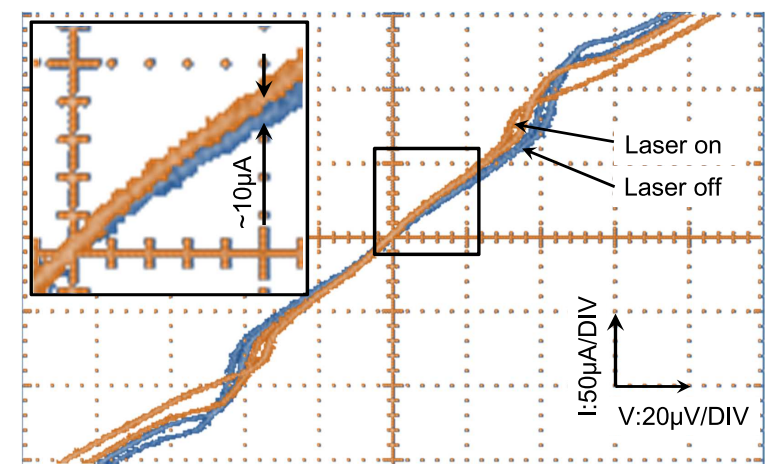

Figure 9: Transition in $I-V$ curves of Hf-STJ with $100 \times 100 \mu \mathrm{m}^{2}$ junction size by continuous illumination of visible laser $(\lambda=465 \mathrm{~nm})$ pulses, measured with 10 Gauss magnetic field at $T=150 \sim 161 \mathrm{mK}$. The $I-V$ curve measured with the laser on is piled up with the $I-V$ curve without the laser. About $10 \mu \mathrm{A}$ increase in the current at $V=20 \mu \mathrm{V}$ is observed for the laser illumination.

as a single photon detector. Finally, developments of readout electronics for Nb/Al-STJs are underway to reduce the readout noise drastically. SOI-STJ is one of the promising candidates among several ultra-low temperature amplifiers under consideration.

\section{Acknowledgment}

We thank Prof. Y. Otsuka at University of Tsukuba for lending us the dilution refrigerator kindly. We also thank KEK Detector Technology Project for supporting us in development of STJs at KEK. This work was supported by the Ministry of Education, Science, Sports and Culture of Japan (MEXT KAKENHI Grant Number 25105007), and the Japan-U.S. cooperative program in high-energy physics.

\section{References}

[1] P. B. Pal and L. Wolfenstein, Phys. Rev. D25 (1982) 766

[2] M. A. B. Beg and W. J. Marciano, Phys. Rev. D17 (1978) 1395

[3] S. H. Kim, K. Takemasa, Y. Takeuchi and S. Matsuura, J. Phys. Soc. Jpn., 81 (2012) $024101 \mathrm{~S}$

[4] S. Matsuura et al., Astrophys. J. 737 (2011) 2

[5] P. A. R. Ade et al. (Planck Collaboration), arXiv:1303.5062 (2013); P. A. R. Ade et al. (Planck Collaboration), arXiv:1303.5076 (2013)

[6] P. Lerch and A. Zehnder, Topics Appl. Phys. 99 (2005) 217-267

[7] M. Kurakado, T. Takahashi and A. Matsumura, Appl. Phys. Lett. 57 (1990) 1933; N. Rando et al., Nucl. Instr. Meth. Phys. Res. A313 (1992) 173

[8] A. Peacock et al., Nature 381 (1996) 135; P. Verhoeve et al., IEEE Trans. Appl. Supercond. 7 (1997) 3359; P. Verhoeve et al., J. Appl. Phys. 83 (1998) 6118; S. Shiki et al., PASJ 56 (2004) L19

[9] K. Kasahara et al., PoS(TIPP2014) 074, Proceeding of TIPP2014 on the talk given on Jun 6th, 2014

[10] S. H. Kim et al., Physics Procedia 37 (2012) 667-674 (Proceedings of TIPP2011); S. H. Kim et al., JPS Conf. Proc. 1 (2014) 013127 